Focused on Russian mechanics contributions for Structural Integrity

\title{
Multi-level model describing phase transformations of polycrystalline materials under thermo-mechanical impacts
}

\author{
Peter Trusov, Elena Makarevich, Nikita Kondratev \\ Perm National Research Polytechnic University, Russia \\ tpv@matmod.pstu.ac.ru, bttp:/ /orcid.org/0000-0001-8997-5493 \\ makareviches@inbox.ru \\ kondratevns@gmail.com, bttp://orcid.org/0000-0002-0261-3017
}

\begin{abstract}
The problem of constructing the multilevel physical model of inelastic deformation in steels allowing to take into consideration diffusionless solid-state phase (martensitic) transitions is considered. The model structure includes three scale levels with the closed system of equations offered for them. Explicit internal variables reflecting the evolution of the material structure (both the defect structure and the grain one) are introduced at the lower scale levels of the model. The distinctive feature of the developed model is consideration of the lower scale level in such a way that a homogeneous element of this level completely turns into a new phase at a high speed (relative to the kinematic quasi-static loading), that is close to the speed of sound in the crystal medium. Based on the principles of classical thermodynamics the phase transformation criterion is written. According to this criterion, the choice of a transformational system under the martensitic transition is made. The algorithm of the model is developed and its realization features are described in connection with the high-rate restructuring of the face-centered cubic lattice to the body-centered tetragonal one. The result of this restructuring is a severe change in the physic-mechanical properties of the material.
\end{abstract}

KEYWORDS. Crystal plasticity; multiscale modeling; phase transformations; microstructure; defect structure; improved steels.

\begin{abstract}
OPEN $\bigcirc$ ACCESS
Citation: Trusov, P., Makarevich, E., Kondratev, N., Multi-level model describing phase transformations of polycrystalline materials under thermo-mechanical impacts, Frattura ed Integrità Strutturale, 49 (2019) 125-139.

Received: 31.03 .2019

Accepted: 16.05.2019

Published: 01.07.2019

Copyright: (C) 2019 This is an open access article under the terms of the CC-BY 4.0, which permits unrestricted use, distribution, and reproduction in any medium, provided the original author and source are credited.
\end{abstract}

\section{INTRODUCTION}

A dvanced high-strength steels have a wide range of applications in industry and technology, which is constantly increasing due to the excellent combination of their plastic and durability properties [1-2]. Enhanced physics and mechanical properties of steels are achieved by the state of grain and defect structure formed as a result of previous thermo-mechanical processing, mechanisms of its formation and evolution [3]. Numerous experimental studies indicate that phase transformations may be the main reason for the set of physical and mechanical properties in steels, ensuring their 
wide usage [4-5]. All known phase transitions for solid state are observed in steels and their alloys, such as polymorphic transformations with a wide range of morphological and kinetic features, eutectoidal (pearlite) transformations, decay of solid interstitial and substitutional solutions, ordering with a change in the local and long-range order in austenite and martensite. The possibility of realization for certain phase transformations and kinetics of transformations depends on the steel composition and the parameters of thermo-mechanical impacts, such as temperature, heating or cooling conditions, holding time, mechanical loading parameters, etc. The important feature of such systems is that the diffusion mobility of metal atoms and carbon is sharply different. Therefore, the crystal lattice restructuring during transformations can occur together with the diffusion redistribution of carbon and alloying elements. Another feature of steels is that during phase transitions of supercooled austenite, the transformation of a face-centered cubic crystal lattice into a body-centered tetragonal lattice can occur simultaneously with the diffusion redistribution of carbon and alloying elements. The experimental study of this issue is quite resource-expensive. Therefore, in solid mechanics, the problem of constructing the models describing the state and evolution of the structure for a material taking into consideration solid-state phase transformations becomes actual. It is widely known, that the physic-mechanical properties of polycrystalline materials and the functional characteristics of finished products are determined by the current state of the structure at various scale levels [6-7]. The latter significantly changes in thermo-mechanical processing of metals. The correct description of the internal structure for a material provides a fundamental opportunity to optimize existing and develop new methods for obtaining materials and products made of them with increased strength and performance characteristics. As a result, in recent decades, models based on explicit considering the mechanisms and the carriers of inelastic deformation (crystal plasticity based multilevel models of inelastic deformation) are of great interest in solid mechanics [8]. As a rule, in such models, the correct description of the existing mechanisms for inelastic deformation requires the introduction of several (two or more) scale levels. The multilevel approach from the point of view of physical description of the occurring processes is rather universal and can be applied to designing structures made of new, not yet existing materials and creating technologies for their manufacturing. The theoretical basis of this approach to the study of inelastic deformation is the methods of mathematical modeling with the introduction of internal variables, supplemented with the model identification and verification procedures. Internal variables make it possible to explicitly include a description of the physical mechanisms, their carriers, and processes accompanying inelastic deformation at various scale levels of a material. Also, the internal variables of a model reflect structural interactions and restructuring the meso- and microstructure of a material. The scale levels involved into consideration are determined by the objectives of the study and the most important mechanisms of inelastic deformation. At the lower scale levels there is a principal possibility for correct accounting the physical mechanisms of inelastic deformation. Thermo-mechanical effects are transmitted from the macro level to the lower scales and cause changes in the internal structure. In turn, the latter determines the effective characteristics of the material at the macro level. In the framework of the multilevel approach to describe inelastic deformation of metals under thermo-mechanical processing a material point with a necessary set of homogeneous (averaged) characteristics is allocated at the macro-level. A set of homogeneous areas corresponds to this material point at one or several lower scale levels. The multilevel approach allows to describe the response of the material with the constitutive relations of a same type at various scale levels. In the framework of this work, Hooke's law in the rate relaxation form, written in terms of asymmetric measures of strain rates, is used. At the lower scale level, crystallite (a homogeneous part of a polycrystalline material) is considered. Each crystallite has a set of properties: anisotropic elastic modules, lattice orientation, a set of slip systems, transformation systems, the thermal conductivity coefficients. In the models of this type, an important aspect is the correct description of the internal variables evolution being responsible for the properties of both a crystallite and a polycrystal [6]. Constitutive and kinematic equations describing the irreversible deformation at the meso-level due to the slip of dislocations, phase transitions, the evolution equations for critical shear stresses (by different mechanisms), description of rotation for the crystallites, the influence of the temperature changing and the attached stresses on the evolution of the defect structure are included into consideration. The problem of taking into account the geometric nonlinearity at the upper scale level and the connection for the similar characteristics of the scale levels remains important [9].

\section{MODELING THE PROCESSES OF INELASTIC DEFORMATION TAKING INTO CONSIDERATION PHASE TRANSFORMATIONS}

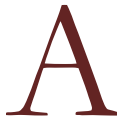

$\mathrm{t}$ present, in scientific literature there are various models of different types to describe the behavior of steels taking into consideration martensite transformations. The reviews of the works devoted to this problem can be found in the articles [10-11]. Two main approaches to constructing the models of polymorphic transformations can been distinguished. The first one is based on the models with explicit account of the phase boundaries taking into consideration 
the conditions at the phase boundary of the deformable material and the kinetics of a new phase evolution. The second one is based on the models with introduction of additional state parameters or model variables characterizing certain features of the material structure "on average" (for example, the concentration of a new phase), and the formulation of relations for them. In the models of the first type, with the explicit introduction of interphase boundaries into consideration [see 12-13], there is an opportunity to describe phase transformations from the point of solid mechanics using the ideas of the classical phase transitions theory by J. Gibbs. Phase boundaries appear in solids as a result of phase transitions. They can be considered as surfaces where deformations have a discontinuity whereas a field of displacement is continuous. The microstructure of a material changing in the process of the phase transition generates its own transformation deformations and modification in the elastic modules. So that, at the phase boundary some components of the strain tensor can break, and it leads to limitations on the constitutive relations. Appearance of an equilibrium discontinuous deformation field in an elastic body requires some regions in the deformation space where the Hadamard's inequality, being a necessary condition for stability with respect to infinitely small deformations, is failed. To maintain an equilibrium at the phase boundary, the following conditions must be satisfied: continuity keeping, force continuity, and a thermodynamic condition being an analogous of the chemical potentials' equality in the Gibbs theory. The latter condition imposes restrictions on determining the shape of the phase boundary and the corresponding deformations at the boundary. Since, even if the defining relations allow the existence of two-phased states, not all deformations can be realized at the phase boundary. This leads to the concept of a phase transitions' zone, which boundary determines the limit surface of transformation in the space of deformations.

For the second type models, the phase field method is often applied, which is used to describe both diffusion transformations and diffusionless (martensite) ones at the meso-level (the simulated area consists of several grains) in many cases [see, for example, 14]. This approach assumes the presence of a "blurred" ("diffusion") boundary between the phases in contrast to the classical methods using the concept of "sharp boundary", when the multiphase structure is described by the position of the boundary and the set of differential equations is solved together with the flow equations and the constitutive equations at the boundary for each of the areas. In the "diffusion boundary" approach, the form and mutual arrangement of the regions occupied by individual phases are described by a set of parameters determining their fraction $\varphi_{i}$. The value of the parameter can vary from 0 to $1 ; \varphi_{i}=0$ corresponds to the area where there is no phase $i, \varphi_{i}=1$ corresponds to the single-phase region. Thus, the microstructure (with the exception of grain boundaries, defects, etc.) can be described by a set of single-phased regions separated by boundaries where more than one value $\varphi_{i}$ is different from zero. In the "diffusion boundary" approach, the change in the shape of the regions (and hence, the position of the boundary) over time is implicitly determined by the change in the fractions of phases. The time change of the phase fractions is described by the kinetic equation obtained in terms of thermodynamics of irreversible processes, i.e. the linear relationship between the rate of change for the phase fractions and the derivative of the thermodynamic potential for this parameter is used. Phase transformations occurring in isothermal conditions are most often investigated, and free energy is taken as a thermodynamic potential, but there are works studying non-isothermal processes where entropy is chosen as a thermodynamic potential [15].

In most studies devoted to description of thermo-mechanical processes, the so-called direct models of the first type are used [16] when a set of finite elements is matched to each grain and a model is used to describe the phase transitions for each of the elements. The usage of such models for modeling the real processes in three-dimensional formulation requires significant computational resources. Therefore, the statistic type models [17] are actual, where the set of homogeneous elements of the lower scale level constitutes a representative volume with homogeneous properties at a higher scale. Within this paper, a multilevel model of the hybrid type to describe the behavior of steels under thermo-mechanical loading, taking into consideration the phase transformations, is proposed by the authors. Within the framework of this model at the macro level, a direct type model is used. To determine the response of each material point at this scale level, a statistic model is applied, comprising the elements from a lower scale. The structure of this model includes internal variables being divided into two groups: explicit ones and implicit ones. Explicit internal variables are included into the constitutive relations at the considered scale level, and the implicit ones are the parameters of evolution equations. To connect the internal variables of two above mentioned groups, the closing equations are applied. When using the approach with explicit introduction of internal variables, the following hypothesis is accepted. The reaction of the material at any moment is determined by the current values of thermo-mechanical characteristics, internal variables and parameters of external influence. The considered hypothesis allows to give up rather complicated constitutive relations in the operator form. At the same time, the material memory about the prehistory of influences is preserved due to the evolving internal variables being the impact history carriers in this case. Within this article, the structure of the proposed model is described, its scale levels are introduced, the constitutive relations and evolutionary equations are given, as well as the algorithm for the implementation of the model. 


\section{THE STRUCTURE OF MULTI-SCALE MODEL WITH PHASE TRANSFORMATIONS}

A solid phase transformation in a polycrystalline material is understood as a polymorphic transformation leading to changing the physic-mechanical properties of some region in the material at a micro- and/or meso-level as a result of the crystal lattice transformation under external influences (loading, temperature, etc.). A phase is understood as some part of a grain being characterized by a specific type of crystal lattice, a chemical composition, a type of solid solutions, etc., at a fixed moment of a thermo-mechanical loading process. From the point of mathematical modeling and solid mechanics a phase is understood as a certain sub-region inside a material which behavior under deformation is described by the constitutive relations of the fixed type with a specified (determined from the solution of some auxiliary subtasks) set of properties being defined by the parameters and the current value of internal variables.

With a goal of modeling the phase transformations of polycrystalline materials in thermo-mechanical processing the multilevel model of a hybrid type is developed. Within this model three structure-scale levels are taken into consideration inside the material. They are macro-level, meso-level I and meso-level II. Internal variables are added into the structure of the model at each scale level being the carriers of impacts' history. The macro-level is supposed to be the material representative volume (consisting of some hundreds of grains). To analyze a behavior of this volume, the boundary value problem is offered to be formulated and solved (for the chosen computational domain) with determination of fields for stresses, strains and temperature in the considered material volume. The finite element method procedure is applied for the numerical solution of the problem at the macro-level. Within the framework of the proposed multilevel model the problem of determining the reaction of a material to the applied thermo-mechanical impact is essentially nonlinear. A step-by-step procedure (in time) is used to solve it. Decomposition of the whole problem according to the physical processes is realized. The subtasks of determining the stress-strain state, the temperature and the problem of determining the phase composition of a material are considered being connected (using a step-by-step procedure). The solution of this problem allows to determine the impacts (velocity gradient, temperature and temperature rate of change) at each point of the considered area (i.e. within each finite element), which are then given down to a deeper scale level within the multilevel model. Thus, within the framework of the finite element scheme at the macro level, the finite elements themselves are precisely the elements of meso-I. As a basic constitutive relation at the meso-I, the generalized Hooke's law in the rate relaxation form and the heat equation are used. The mentioned constitutive relations contain in their structure explicit internal variables. The values of those variables depend on the history of the impacts on the material, are changed in the process of deformation and are determined from the deeper scale levels as a response to the thermo-mechanical effects. For example, the tensor of elastic properties of a material, the inelastic strain rate tensor, the heat capacity coefficient, the thermal conductivity tensor, the power of internal heat sources can be such variables. Herewith, an element of the meso-I is understood as a certain subdomain of a grain, the state of which at each moment of the thermo-mechanical loading process is assumed to be homogeneous in all parameters characterizing the state of the meso-I element, and within which the crystal lattice of the material can be considered as approximately perfect (a grain is supposed to consist of crystallites with a minor misorientation). In turn, a meso-I element is represented as an aggregate, consisting of $\mathrm{N}$ elements of meso-II, such as subgrains, fragments, cells, phase components. Wherein, to determine the response of the meso-I element, the modified statistical model (taking into account the relative position of the neighboring meso-II elements) is used [17]. Herewith, a size of a meso-II element is assumed to be so small that its state can be considered as a homogeneous one according to all parameters at each time moment. The velocity gradient, temperature, and the rate of temperature changing are transmitted from meso-I to meso-II as impact factors. A model based on crystal plasticity is applied for the meso-II element. The Voigt hypothesis is used when transmitting exposure from meso-I to meso-II. At any fixed moment of a thermo-mechanical loading process each meso-II element is supposed to be in some specific phase (i.e. it is always single phased), but the phase characterizing it can change as a result of external influence, which leads to a change in all its properties. Belonging to a particular phase determines the basic properties of the meso-II element, including the type of its crystal lattice. Orientation of the axes of the moving coordinate system [18-19] is considered as known for each meso-II element. This orientation changes during a deformation process (as a result of rotation for the meso-II element or as a result of the transformation the element lattice after the realized phase transition). The moving coordinate system is associated with the lattice of the element (but, doesn't coincide with it in general case, as the lattice may have distortions in the deformation process). As a result of thermal and mechanical effects (transmitted from the upper scale level) in case of fulfillment the thermodynamic criterion a phase transition can occur in a meso-II element. Herewith, due to the homogeneity of the all parameters' values for the meso-II element, it is assumed that its entire volume undergoes a phase transformation simultaneously. All processes in the meso-II element are considered in its moving coordinate system being oriented definitely with respect to the laboratory coordinate system. Wherein, the following modes are realized in the meso-II element: inelastic deformation by 
shears on slip systems, the lattice rotation i.e. quasi-rigid motion with a spin tensor being determined by one or another rotation law (here, the Taylor model is used), temperature deformation, deformations of an element caused by a phase transformation, elastic distortions of a crystal lattice in a crystallite, the changing the lattice type of the crystallite and all its properties (internal variables) as a result of phase transformation, the changing the orientation of the moving coordinate system for the element as a result of a phase transformation, the formation of internal heat sources as a result of inelastic deformation processes and phase transformations at meso-II level. The acting stresses in the element, the inelastic strain rate tensor, the power of internal heat sources (due to latent heat of phase transformations and inelastic deformation), the orientation tensor of a meso-II element, characterizing the current orientation of the moving coordinate system for the element with respect to the fixed laboratory coordinate system, and the information about phase composition are transmitted as a response from meso-II to meso-I. The statistical averaging procedures are carried out at the meso-I level (among all meso-II elements, taking into consideration the current orientations of the moving coordinate systems for the elements) to determine the values of the model internal variables at an integration point for a finite element (for simplexelements, this matches the data for an entire finite element). Thus, the meso-I element constitutive relations are "formed" in the process of solving the problem depending on transformation of the material structure at a deeper scale level in the thermo-mechanical processing and may change during the process itself. The constitutive relations constructed by this way for the meso-I element are then used to solve the boundary value problem at the macro level at the next time step.

The subject for discussion in this article is a description of the structure and the implementation features of the model at the meso-levels I and II.

\section{About motion decomposition for the meso-II element}

As in solving the boundary value problems connected with description of thermo-mechanical processing for polycrystalline materials, as a rule, it is necessary to take into consideration large displacement gradients, the problem under consideration, being essentially nonlinear, is posed and solved in a rate form. Within the framework of the model, description of kinematics for the meso-II element is based on introduction of the multiplicative decomposition for the deformation gradient by the following way:

$$
\mathbf{f} \equiv \mathbf{f}^{e} \cdot \mathbf{f}^{t r} \cdot \mathbf{f}^{\theta} \cdot \mathbf{f}^{p}=\overline{\mathbf{f}}^{\mathrm{e}} \cdot \mathbf{r} \cdot \mathbf{f}^{t r} \cdot \mathbf{f}^{\theta} \cdot \mathbf{f}^{p}
$$

where $\mathbf{f}^{e}, \mathbf{f}^{t r}, \mathbf{f}^{\theta}, \mathbf{f}^{p}$ are elastic, transformation, temperature and plastic components of the deformation gradient; $\overline{\mathbf{f}}^{e}$ is the component of the deformation gradient characterizing the elastic distortion of the lattice with respect to the rigid moving coordinate system connected with the lattice of the initial phase; $\mathbf{r}$ is the rotation tensor describing the material rotation together with the moving coordinate system (from the initial position of the moving coordinate system to its current position); $\mathbf{f}^{p}$ is the plastic component of the deformation gradient that does not change the symmetry properties of the material. Based on the accepted decomposition (1) the transposed velocity gradient is defined as follows:

$$
\begin{aligned}
& \mathbf{1}=\dot{\mathbf{f}} \cdot \mathbf{f}{ }^{-1}=\dot{\overline{\mathbf{f}}}^{e} \cdot \overline{\mathbf{f}}^{e-1}+\overline{\mathbf{f}}^{e} \cdot \dot{\mathbf{r}} \cdot \mathbf{r}^{T} \cdot \overline{\mathbf{f}}^{e-1}+\overline{\mathbf{f}}^{e} \cdot \mathbf{r} \cdot \dot{\mathbf{f}}^{t r} \cdot \mathbf{f}^{t r-1} \cdot \mathbf{r}^{T} \cdot \overline{\mathbf{f}}^{e-1}+ \\
& +\overline{\mathbf{f}}^{e} \cdot \mathbf{r} \cdot \mathbf{f}^{t r} \cdot \dot{\mathbf{f}}^{\theta} \cdot \mathbf{f}^{\theta-1} \cdot \mathbf{f}^{t r-1} \cdot \mathbf{r}^{T} \cdot \overline{\mathbf{f}}^{e-1}+\overline{\mathbf{f}}^{e} \cdot \mathbf{r} \cdot \mathbf{f}^{t r} \cdot \mathbf{f}^{\theta} \cdot \dot{\mathbf{f}}^{p} \cdot \mathbf{f}^{p-1} \cdot \mathbf{f}^{\theta-1} \cdot \mathbf{f}^{t r-1} \cdot \mathbf{r}^{T} \cdot \overline{\mathbf{f}}^{e-1}
\end{aligned}
$$

When considering metallic polycrystals, the magnitude of the elastic distortions of the lattice can be assumed to be small, therefore $\overline{\mathbf{f}}^{e} \approx \mathbf{I}$ and the relation (2) is converted into the following form:

$$
\begin{aligned}
\mathbf{1}=\dot{\mathbf{f}} \cdot \mathbf{f} & =\dot{\overline{\mathbf{f}}}^{e} \cdot \overline{\mathbf{f}}^{e-1}+\dot{\mathbf{r}} \cdot \mathbf{r}^{T}+\mathbf{r} \cdot \dot{\mathbf{f}}^{t r} \cdot \mathbf{f}^{t r-1} \cdot \mathbf{r}^{T}+\mathbf{r} \cdot \mathbf{f}^{t r} \cdot \dot{\mathbf{f}}^{\theta} \cdot \mathbf{f}^{\theta-1} \cdot \mathbf{f}^{t r-1} \cdot \mathbf{r}^{T}+ \\
& +\mathbf{r} \cdot \mathbf{f}^{t r} \cdot \mathbf{f}^{\theta} \cdot \dot{\mathbf{f}}^{p} \cdot \mathbf{f}^{p-1} \cdot \mathbf{f}^{\theta-1} \cdot \mathbf{f}^{t r-1} \cdot \mathbf{r}^{T}
\end{aligned}
$$

Then, the additive decomposition of the transposed velocity gradient in the actual configuration based on the multiplicative decomposition (1) can be represented as follows

$$
1=\overline{\mathbf{1}}^{\ominus}+\omega+\mathbf{1}^{t r}+\mathbf{1}^{\theta}+\mathbf{1}^{p},
$$


where $\omega=\dot{\mathbf{r}} \cdot \mathbf{r}^{T}$ is the spin determining the rate of rotation for the moving coordinate system connected with the material symmetry axes of a meso-II element, $\overline{\mathbf{1}}^{e}=\dot{\overline{\mathbf{f}}}^{e} \cdot \overline{\mathbf{f}}^{e-1}$ is the rate of elastic distortions of the lattice.

\section{The model constitutive relations for a meso-II element}

As the model is intended to describe the processes of thermo-mechanical treatment being characterized by large displacement gradients, geometrically nonlinear kinematic and constitutive relations are used in its structure [18-19]. The rate statement of the problem is done in the current configuration and the following constitutive relations are used [20-21]:

$$
\mathbf{k}^{c r}=\Pi:\left(1-\omega-1^{p}-1^{\theta}-\mathbf{1}^{t r}\right) ; \quad \mathbf{k}^{c r}=\dot{\mathbf{k}}+\mathbf{k} \cdot \omega+\omega^{T} \cdot \mathbf{k} ; \quad \mathbf{k}=\stackrel{\varrho}{\varrho} / \hat{\varrho} \sigma
$$

where $\Pi$ is the tensor of elastic properties for the meso-II element, defined by the constant components in the basis of the crystallographic coordinate system of the current phase in the initial configuration; $\mathbf{1}=\mathbf{v} \hat{\nabla}$ is the transposed velocity gradient for the material particles of the meso-II element in the current configuration, transmitted from the meso-I; $\mathbf{1}^{p}$ is the plastic part of the relative velocity gradient connected with the shears on the slip systems inside the meso-II element in the deformation process; $\mathbf{1}^{\theta}$ is the thermal part of the transposed relative velocity gradient; $\mathbf{1}^{\text {tr }}$ is the transposed velocity gradient of the transformation deformation, associated with the phase transformation in the material; $\mathbf{k}, \mathbf{k}^{\text {cr }}$ are the weighted Kirchhoff stress tensor and its corotational derivative; $\stackrel{\circ}{\varrho}, \hat{\varrho}$ are the densities of the meso-II element's material in the initial (unloaded) and current configurations (the density depends on the phase the element at the considered moment is in); $\sigma$ is the Cauchy stress tensor of the meso-II element; $\boldsymbol{\omega}$ is the spin tensor of the meso-II element (to define it, any physically based model of rotation can be used, for example, it can be the Teylor's model of turning in a fully constrained conditions [22] or the model of lattice rotation [18]). Wherein, at each moment of the process, the rotation of the rigid moving coordinate system's axes of the meso-II element, connected with the lattice of the element in its current phase, is considered. The plastic part of the velocity gradient $\mathbf{1}^{p}$ is determined by shears on the slip systems in the meso-II element:

$$
\mathbf{1}^{p}=\sum_{k=1}^{K} \mathbf{b}^{(k)} \mathbf{n}^{(k)} \dot{\gamma}^{(k)}
$$

where $k$ is the number of the slip system. Herewith, the shear rate on each slip system in the meso-II element is considered as a function of the acting stresses $\tau^{(k)}$, critical shear stresses $\tau_{c}^{(k)}$ and temperature $\theta$ :

$$
\dot{\gamma}^{(k)}=f\left(\tau^{(k)}, \tau_{c}^{(k)}, \theta\right)
$$

in general case, any physically valid model can be used to define it. In particular, a non-linear viscoplastic model [21, 23] can be used:

$$
\dot{\gamma}^{(k)}=\dot{\gamma}_{0}\left(\frac{\tau^{(k)}}{\tau_{c}^{(k)}}\right)^{1 / m} H\left(\tau^{(k)}-\tau_{c}^{(k)}\right) .
$$

The thermal part of the transposed relative velocity gradient in the meso-II element is defined using the following relation:

$$
1^{\theta}=\dot{\theta} \alpha,
$$

where $\alpha$ is the thermal expansion tensor for the material of the meso-II element. Herewith, a simplification can be accepted for cubic crystals, and the relation for the thermal component of the velocity gradient can be written in the following form: 


$$
\mathbf{1}^{\theta}=\alpha \dot{\theta} \mathbf{I}
$$

where $\alpha$ is the coefficient of linear thermal elongation-compression for the material of the meso-II element, $\mathbf{I}$ is the unit tensor. The power of internal heat sources in the meso-II element is determined by two components connected with, firstly, the inelastic deformation on the slip systems in the element, and secondly, the phase transformation in the meso-II element:

$$
q_{\text {meso }}=\sum_{k=1}^{K} \tau^{(k)} \dot{\gamma}^{(k)}+\hat{\varrho} g \dot{\xi}
$$

where $g$ is the specific latent heat of a phase transition, $\dot{\xi}$ is the new phase (martensite) formation rate.

\section{Determining the transformational part of the deformation gradient at the meso-II}

Martensitic transformations occur at speeds comparable to the speed of sound in the crystal environment under consideration. In multilevel models, as a rule, the martensitic phase transitions are considered at a higher scale level [for example, 24], than the meso-II one (according to the division of the scale levels adopted in this work). It allows to use the magnitude of the martensite volume fraction in the considered volume to describe phase transitions, "smoothing" and assuming it as a continuous value in the process of thermo-mechanical loading, i.e. the martensitic transformation process is "smeared" in time and space. Within the framework of this model, the meso-II element is assumed to be so small, that it turns into a new phase almost instantly. Wherein, out of all possible variants of the transformational system, an energetically more favorable one is chosen. The hypothesis is accepted that the phase transition is fully realized in a time step (in the general algorithm of the inelastic deformation model). For the elements experiencing a phase transition at a given time step, a time step is divided into a fixed number of substeps. The number of substeps is determined in numerical experiments. For these elements experiencing a phase transition, the temperature changing per step is neglected; the velocity gradient is assumed to be fixed throughout the entire step. In this case, the additive decomposition of the transposed velocity gradient in the current configuration, based on the multiplicative decomposition (4), can be represented as:

$$
1=\overline{\mathbf{1}}^{e}+\omega+\mathbf{1}^{t r}+\mathbf{1}^{p},
$$

Within the framework of the proposed model, at this stage, the relations, allowing to determine the gradient of transformational deformation for the martensitic phase transformation obtained in [25-26] based on the application of crystallographic theory of martensitic transformations in steels [27-28], are used. The transformational deformation gradient of the meso-II element during the transformation from the initial phase to the new one under the conditions of deformation with an invariant plane can be represented as follows:

$$
\mathbf{f}^{t r}=\mathbf{I}+\xi \mathbf{s m}
$$

where the vector $\mathbf{m}$ of a normal to the invariant habit plane (a unit vector) and the vector $\mathbf{s}$ of a shear set the transformational system (these vectors are not perpendicular in a general case). These vectors aren't determined by the crystallography entirely, like their analogues in the theory of plastic shear along slip planes. They are calculated taking into consideration the magnitude of changing the lattice parameters during the phase transition and the accommodation mechanisms accompanying it [25]. The volume fraction $\xi$ after a martensitic phase transition within the element, is introduced into consideration. The dependence of the volume fraction $\xi$ on the current time at the step is given by a smooth function, being satisfied by the following properties: 1) at the beginning of the first substep of the phase transition, it is equal to 0 , at the end of the last substep it is equal to $1 ; 2$ ) at the ends of the phase transition interval (time step), the $\xi$ derivative is equal to 0 or 1 . It is necessary to note that the latter requirement is optional. Further, an internal step-by-step process of solving according to the initial scheme of the elastic-viscoplastic problem is organized for these elements. Wherein, the three components of the deformation gradient are changed: the elastic one, the transformational one and the viscoplastic one. Herewith, the critical shear stress is determined by averaging the critical austenite stress (with a weight (1$\xi)$ ) and the critical martensite one (with a weight $\xi$ ). The transformational component of the relative velocity gradient $\mathbf{1}^{\text {tr }}$ is determined by the transformational deformation gradient, being depended on the phase transformation type occurring in the material, and has the following form: 


$$
\mathbf{1}^{t r}=\dot{\mathbf{f}}^{t r} \cdot \mathbf{f}^{t r-1}=\frac{\dot{\xi}}{\operatorname{det}\left(\mathbf{f}^{t r}\right)} \mathbf{s m}
$$

\section{CHOOSING THE PHASE TRANSFORMATION CRITERION FOR A MESO-II ELEMENT}

I $\mathrm{n}$ researches on phase transformation modeling different approaches for creating the relations to determine the volume fraction of a new phase in a material under consideration depends on the impact process parameters are used. The Koistinen-Marburger [29] type equations are the most widely used ones in material science practice in case of macrophenomenological description of heat treatment processing. The relations of such a type and their different modifications make it relatively easy to determine parameters in equations for phases' volume fractions from the macro-experiments. But these relations don't allow to take into consideration the physics peculiarities in the process of a new phase forming at the meso-scale and don't allow to include besides the temperature impact also the mechanical one into consideration. They also naturally don't allow to analyze the influence of a thermo-mechanical impact parameters on the process of transformation. In recent decades, the alternative thermodynamic approach to the constructing the kinetic equations for the description of the phase volume fractions evolution and the phase transformations criteria has been evolved. This approach allows to construct the models for continuum media and suggest the relations for thermodynamic driving forces of phase transformations based on the analyses of the nature of the effects on the material and the physic-mechanical processes occurring inside the structure of the material during these effects.

\section{The short review of the thermodynamic criteria of phase transformations}

Nowadays, there is a significant amount of papers wherein exactly the thermodynamic approach is used to construct a phase transformation criterion. The short overview for the ways of introducing this criterion in some of them is given below.

In the paper [30] the double phased system (austenite + martensite) is considered as a thermodynamic nonequilibrium system with dissipation. It is supposed that the principle of maximum dissipation can be used to describe its evolution. Herewith, the dissipation power is determined as a difference between the power of external forces and the Helmholtz free energy rate of change for the quasistatic isothermal case considered in the cited article. The specific Helmholtz free energy (per unit volume) is supposed to be consisted of three parts: free chemical energy, surface energy and elastic strain energy. An increment of free chemical energy is expressed by the difference between the corresponding free chemical energies of martensite and austenite being multiplied by the volume fraction of martensite. It is assumed to be a linear function of temperature. Elastic energy is defined as convolution of the stress and the difference between total and phase deformations. All the values are determined at the meso-scale. The change in free energy associated with the boundaries is introduced through the analyses of jumps in chemical and elastic energy and boundary motion speed. The term associated with discontinuities at the phase interface also appears in the power of external forces. In terms of the specified parameters, an expression for the dissipation power is obtained. The force criterion (analogically with plastic deformation) is used as a condition of phase transformation realization. The critical stresses of phase transformations are supposed to be linearly related to the accumulated shifts in austenite (moreover, the coupling coefficient is negative, i.e. the accumulated shifts in austenite make the critical phase transformation stresses lower) and the martensite volume fraction. In [31] the relation for dissipated energy with excretion the generalized thermodynamic force of phase transformation (being conjugate to the rate of change for the specific volume fraction of the martensite phase) explicitly is given. The specific relations for the thermodynamic forces under various conditions (for example, the absence of internal additional stresses in austenite from phase transformation) are considered. Separately, the definition of the thermodynamic force associated with the inhomogeneous plastic deformations in the austenitic phase is considered. Herewith, the inhomogeneous plastic deformations are assumed to be proportional to the average plastic deformations in austenite.

In the paper [32] it is suggested to determine the generalized thermodynamic force using the difference of the Helmholtz free energy in the initial and martensite phases and the meso-stresses' work averaged over two phases made on the difference between transformation and plastic deformations in the initial and final phases. In turn, the Helmholtz free energy is represented as a sum of "chemical" energy (determined by the position of the atoms in the phases and dependent on temperature) and strain energy (dependent on elastic strains and temperature). The excess by the generalized thermodynamic force its critical value for a given material is used as a criterion of a possibility for the phase transformation.

In the paper [33] the rate of change for the volume fraction of each variant in martensite phase is determined by the power low depending on the shear stress in the habit plane of the transformation and hydrostatic stress. To determine the critical 
resistant stress to the martensite transformation, the Olson's and Roitburd's solution, based on the assumption of the spherical shape of the original grain, is used. For the martensite plate the shape of a flattened ellipsoid is taken. Into the proposed relation, there is chemical energy difference (the Gibbs free energy), surface energy of an "austenite - martensite" boundary and transformation strain energy.

In [34] to describe the martensite transformation, a set of internal variables is introduced (the martensite volume fractions for each of the possible variants). Their associated thermodynamic forces are introduced being dimensioned as energy per unit volume. The dissipation rate due to the phase transition has been defined as the sum of multiplications of thermodynamic forces and the rate of change for the martensite fraction (in all variants). The thermodynamic forces are dependent on elastic energy, temperature, crystallography of the martensite transformation, the fraction of the transformed phase (according to different variants). The criterion for implementation of the phase transformation is also the excess of the critical value by the chosen thermodynamic force.

In [35], following the model proposed by Olson and Cohen [36], temperature and hydrostatic stress (related to the stress intensity) are considered to be the driving forces of the transformation; plastic deformations of the austenitic phase are taken into account; it is believed that martensite nuclei are formed by the intersection of the shear bands.

In [37-38] an expression for the thermodynamic potential is introduced. As a potential the Gibbs free energy is used. Constitutive relations (for strains and entropy) are obtained from the second law of thermodynamics. The expressions for the dissipation rate, the thermodynamic force conjugated with a fraction of the martensite phase, and the temperature for the onset of the martensite transformation (taking into account the effective stresses) are given. The formulations for the corresponding evolutionary equations are given.

The paper [24] contains a thermodynamic description of the phase transformation process, based on Onsager's (linear) irreversible thermodynamics. In accordance with the Onsager formalism, dissipation is represented as a sum of the products of affinity (essentially, thermodynamic forces) and flows. The hypothesis of additive decomposition for entropy into reversible (elastic) and irreversible (transformational) components is accepted. The latter is determined through the latent heat of a phase transformation. The expressions for thermodynamic forces are obtained from the 2nd law of thermodynamics in the form of dissipation inequality using different thermodynamic potentials such as internal energy, the Helmholtz and the Gibbs free energies. The achievement of the corresponding critical value by the thermodynamic force on the transformation system is taken as the criterion of phase transformation.

The paper [39] contains thermodynamic analysis of phase transformation. In particular, the relations for the components of free energy (Helmholtz) for the representative volume and energy dissipation are given. Free energy has been defined as a sum of elastic strains energy, crystallographic energy (often called chemical energy) and energy of interphase boundaries (the latter can be neglected, as well as energy of the defects (point, dislocations, etc.)). Elastic energy is decomposed into elastic energy of the average (for a representative volume) elastic strains and energy of interphase interactions. To determine the latter, analytical solution in terms of the Green function and the Eshelby tensor is applied. Using the Legendre transformation transition from the Helmholtz free energy to the Gibbs free energy is realized. The latter is completed with restriction on the total fraction of the phases and the non-negativity condition for the fraction of the phase for each variant using Lagrange multipliers method and Kuhn-Tucker conditions. The expression for internal dissipation, determined by the difference between the power of external forces and the rate of change for free energy is obtained. From it, the relation for the driving (thermodynamic) force is derived to determine the phase boundary displacement.

Paper [40] provides relations for determination the "driving mechanical force of transformation" (in fact, the work of applied stresses on transformation strains), elastic stored energy of the environment, and dissipated plastic work of deformations. At the same time, for a specific implementation, the criterion of accumulation of a certain plastic strain level in the residual austenite is used as a condition for a phase transformation.

In [41], additive decomposition of specific (per mass unit) entropy into elastic (reversible), plastic and transformation parts is introduced. The transformation part is determined by the weighted sum of the latent heat of phase transformations according to different variants divided by phase transition temperature. The rate of change for the plastic part, related only to austenite, is determined by the sum of multiplications of the entropy change measure due to the shifts in each slip system and the shift rate in the corresponding systems. Following by the procedure proposed by Coleman and Noll, the dissipation function is introduced being represented by the sum of multiplications for thermodynamic forces and flows. A general form of the defining relation and expression for thermodynamic forces is obtained from the dissipation inequality. From the internal energy (included into the dissipation expression), the transition to the Helmholtz free energy is made. The Helmholtz free energy is proposed to be written as a sum of the following components: elastic, thermal (including the heat of phase transformations), surface and energy of defects (dislocations). Specific expressions for each of the introduced components are proposed. A similar decomposition for the thermodynamic (driving) forces of phase transformations and plastic deformation is proposed. Relations for determination all the components included into the specified decomposition 
are introduced. The kinetic equations for the corresponding flows (fractions of martensite according to the variants and shear rates for slip systems) and the phase transformation criterion (in terms of the thermodynamic force) are formulated. In [42-43], the Helmholtz free energy and the dissipative function are introduced for each of the phases. Free energy is represented as a sum of chemical, elastic and "accumulated plastic" parts. A specific view for each of them is proposed. The dissipative function is determined by the difference between the mechanical work performed on the body and the Helmholtz free energy. The expressions for the driving force and the criterion of phase transformation are also obtained from the dissipation inequality.

In [44] there is a criterion of phase transformations (for direct and reverse ones) including the driving force of the phase transformation (consisting, in turn, of the mechanical (stress) and thermodynamic (determined through the "chemical" potential of components) and the critical value of the driving force. And besides, the latter is adopted to be the same for the forward transformation and the reverse one. At the same time, the critical force of formation for a martensite nucleus is assumed to be higher than its critical value for propagation of the transformation front.

\section{About the phase transformation criterion choosing}

Within the framework of the proposed structure of the model, the formation of the new phase is assumed to occur at the meso-level II. For a fixed time moment, a thermodynamic criterion of a phase transformation is checked out for each element, allowing to choose an active transformation system for a given element from energy considerations, as well as the elements (within a representative volume) being energetically beneficial to move into a new phase under these conditions. In this work, the meso-II element is considered as a closed thermodynamic system which has undergone some influence (temperature and kinematic) at a fixed moment of process. The phase transformation criterion is formulated for the mesoII element and, due to homogeneity of all parameters' values for this element, the hypothesis, that its entire volume goes into a new phase completely if the phase transformation criterion is carried out for it at a fixed moment of process, is accepted. The construction of the phase transformation criterion for an element is based on the approach, used in the work [45], where based on the principles of classical thermodynamics of irreversible processes [46-47], a kinetic equation for a new phase fraction in a certain multiphase volume, has been obtained. The hypothesis about the "single-phased" meso-II element at any arbitrary moment allows to simplify the expression being obtained in the above-cited paper for the thermodynamic driving force of phase transformation and write it in the following form:

$$
\Delta G^{0-p}=G^{0}-G^{p} \geq G^{*} ; \quad G=G\left(\text { phase }, c_{1}, c_{2}, \ldots, \mathbf{q}^{e}, \theta\right),
$$

where $G^{0}$ is the free energy (Gibbs) function of the element in the initial phase; $G^{p}$ is the free energy (Gibbs) function of the element into some phase $p$, whereto the element can (theoretically) go at the current values of the model internal variables under conditions of a given thermo-mechanical effect; $\Delta G^{0-p}$ is the phase transformation driving force; $c_{1}, c_{2}, \ldots$ are the component concentrations; $\mathbf{q}^{e}$ is an elastic strain measure; $\theta$ is temperature. Herewith, free energy of the system is considered to be a function of the current phase characteristics, the component composition, the elastic part of strain measure and temperature. According to the introduced criterion, the phase transition is realized for the meso-II element if the change of the thermodynamic potential value for the system (in this case, it is the Gibbs free energy) during the transformation from the initial phase (index 0) to some new phase (index p) exceeds some critical threshold. It should be noted that within the framework of the proposed model numerical implementation, the values of all internal variables and parameters characterizing the stress-strain state of the meso-II element are changed at the end of a time step within the used computational scheme. Wherein, the verification of the phase transition criterion for each meso-II element is performed at the current step. For this, a special computational procedure with splitting the main numerical algorithm time step into substeps and the "virtual" transfer of the element into a new phase is implemented inside the step. If the phase transition criterion is fulfilled at the end of a step for an element, it is considered to have passed into a new phase and all its properties are redefined. The other elements (for which the criterion of phase transformation was not fulfilled) remain in the initial phase. Details of the algorithm for checking the phase transition criterion fulfillment for the meso-II element are described below in the corresponding section.

The specific free energy of an element in the initial phase is assumed to consist of the specific elastic energy $G^{e}$ and the specific chemical energy $G^{\text {ch }}$ :

$$
G^{0}=G^{e}\left(\text { phase }, \mathbf{q}^{e}, \Pi\right)+G^{c h}\left(\text { phase }, c_{1}, c_{2}, \ldots, \theta\right) \text {. }
$$


The contribution associated with the specific surface energy is not taken into consideration, as the phase boundaries are not explicitly considered within the statistical submodel of this level. Herewith, within the accepted structure of the multilevel model, all properties of the meso-II element (such as the tensor of elastic characteristics, the elastic part of the strain measure, etc.) are defined in its moving coordinate system associated with the lattice, and therefore, are also dependent on the phase the element is in, at the considered moment of the process. The elastic part of the specific free energy is a quadratic form of the elastic part of the strain measure and in the moving coordinate system of the element at the current time moment it can be determined as follows:

$$
G^{e}\left(\text { phase }, \mathbf{q}^{e}, \Pi\right)=\mathbf{q}^{e}: \Pi: \mathbf{q}^{e},
$$

where elastic deformations in the meso-II element are determined as a result of integration of the elastic part of the relative velocity gradient $\overline{\mathbf{1}}^{e}$ at a current moment (i.e., they directly depend on the external action parameters, the type of the element crystalline lattice and the orientation of this lattice at the considered moment of the process), where $\overline{\mathbf{1}}^{e}$ is defined from the decomposition (4) without the transformational part, but with allowance for plastic and temperature deformations in the element at the considered time moment.

It is assumed that the specific free energy of some newly formed phase $p$ can be represented in the following general form:

$$
G^{p}=G^{c b}\left(\text { new_phase, } c_{1}, c_{2}, \ldots, \theta\right)-G^{t r}(\text { new_phase, } \sigma, \theta, \dot{\theta})
$$

where $G^{\text {tr }}$ is a transformational part of the specific free energy associated with implementation of the phase transformation in the system under consideration (the meso-II element). In particular, in simulating a martensitic transformation, the rate of temperature changing is assumed to be so high, that no diffusion processes have time to go through the material; i.e., the component composition of the element remains unchanged. For further describing the diffusion phase transformations, it is necessary to take into consideration the cooling rate and to describe the diffusion processes occurring in the material at the meso-level. Within this work, in describing the diffusionless (martensitic) transformation, the transformational part of the specific free energy is introduced in the following form:

$$
G^{t r}(\text { new_phase, } \sigma)=\sigma: \mathbf{s m}
$$

The chemical component of the meso-II element specific free energy in a certain phase includes both free energy of a separate phase itself and contributions into the free energy due to mixing and chemical interaction of the components. By analogy with the works [48-50], it is determined using the following relation:

$$
G^{c b}\left(\text { phase }, c_{1}, c_{2}, \ldots, \theta\right)=\frac{1}{v} \sum_{k=1}^{n} c_{k} g^{k}(\theta)+\frac{\theta \mathrm{R}}{v} \sum_{k=1}^{n} c_{k} \ln \left(c_{k}\right)+\frac{1}{v} \sum_{k=1}^{n-1} \sum_{j=k+1}^{n} c_{k} c_{j} r^{k j}(\theta),
$$

where $c_{k}$ is a $k$-component concentration in a phase under consideration, measured in mole fraction; $g^{k}$ is a specific free energy of a separate component $k$ in the considered phase; $v=N_{a} v_{c} / z$ is a mole volume (where $N_{a}$ is an Avogadro constant; $v_{c}$ and $z$ are the unit cell volume of the material and the number of atoms per cell in the considered phase, respectively); $r^{k j}$ is a parameter, depending on temperature and describing interaction between the components $k$ and $j$ within the considered phase; $R=k_{B} N_{a}$, where $k_{B}$ is a Boltzmann constant. The free energy of each individual component $g^{k}$ is approximated by a functional dependence on temperature of the following type:

$$
g^{k}(\theta)=\chi_{1}^{k}+\chi_{2}^{k} \theta+\chi_{3}^{k} \theta \ln (\theta)
$$

where $\chi_{1}^{k}, \chi_{2}^{k}, \chi_{3}^{k}$ are the constants determined for the constituent component of the material, depending on the phase it is in, using the thermodynamic databases [51]. 


\section{FEATURES OF THE MODEL IMPLEMENTATION ALGORITHM AT THE MESO-LEVEL}

$\mathrm{D}$ escription of the algorithm general structure for implementing a two-level statistical model without taking into consideration phase transitions can be found, for example, in the paper [52]. The features of the algorithm taking into consideration the phase transformations in the material in the process of external thermo-mechanical impacts are described below.

At the considered moment of the thermo-mechanical loading process, each element of meso-II (within the framework of the modeled representative volume of meso-I) is exposed to the impact from the upper scale level. The velocity gradient $\mathbf{1}=\mathbf{v} \hat{\nabla}$, temperature $\theta$ and the temperature changing rate are transmitted into it. The problem of determining the stressstrain state of a meso-II element is solved in its own moving coordinate system (at each fixed moment of the process it is definitely oriented relative to the laboratory coordinate system). The calculation is carried out at the current (determined at the end of the previous time step) values of all parameters (including the internal variables). The shear rates for all slip systems within the element are calculated using relation (8), being allowed to determine the inelastic part of the relative velocity gradient $\mathbf{1}^{p}$ from Eqn. (6). The spin $\boldsymbol{\omega}$ of an element moving coordinate system is determined using the Taylor rotation model (full constraint). The thermal part of the transposed relative velocity gradient $\mathbf{1}^{\theta}$ is determined from relation (9). Farther, the velocity of lattice elastic distortions for a material in the initial phase is calculated:

$$
\overline{\mathbf{1}}^{e}=1-\omega-1^{p}-\mathbf{1}^{\theta} .
$$

The last relation is integrated to determine the part of the deformation gradient $\overline{\mathbf{f}}^{e}$, characterizing the elastic distortions of the lattice with respect to the current rigid moving coordinate system of the meso-II element. The value of the Kirchhoff weighted stress tensor corotational derivative $\mathbf{k}^{a r}$ is determined from relations (5), and as a result of its integration, the Kirchhoff tensor $\mathbf{k}$ and the Cauchy stress tensor $\boldsymbol{\sigma}$ are defined themselves. Thus, as a result of integrating the found values at the end of the step, stresses and strains acting in the element are defined. The procedure of the stress-strain state determining is performed for all meso-II elements within the framework of the mezo-I representative volume with the definition of the values for all model internal variables at the end of the time step. Farther, a phase transformation at the meso-level II is assumed being able to occur during the considered time step. The verification of the phase transition criterion (15) fulfillment is carried out for all meso-II elements with examining all possible transformation options for each element. In particular, in simulating a martensitic transformation, the meso-II element is assumed being able to experience 24 transformation variants (corresponding to 24 variants of martensite, obtained from the Kurdjumov-Sachs relationships [27-28]). The most energetically favorable transformation option, for which the value of the thermodynamic driving force $\Delta G^{0-p}$ is greater (at the end of the considered time step), is chosen of all the possible ones. To implement the phase transition in the meso-II element within the framework of the adopted calculation scheme, the full time step is divided into a fixed number of substeps (their required number is determined in computational experiments). The element is assumed jumping completely into a new phase in a full time step. Herewith, the smoothing the transition process is realized at the substeps inside the full step, with introducing the fraction of a new growing phase. The process of solving the problem within the time step (at substeps) is carried out without taking into account the changing the temperature component of the impact inside the step, the velocity gradient (full) is determined at the beginning of the full time step and is considered as fixed within the whole step. An internal step-by-step process for solving the elastic-viscoplastic problem (on substeps) is implemented at the time step for each element, taking into account the transformational component of the relative velocity gradient $\mathbf{1}^{\text {tr }}$, defined from relation (14) (wherein, the accommodative mechanisms are realized due to plastic shears). In calculating inside the step the critical shear stress is defined as a result of averaging the critical stresses of the initial (with a weight $(1-\xi)$ ) and the new (with weight $\xi$ ) phase. The value of the element thermodynamic potential used in the energy criterion in a new phase is assumed to be determined at the end of the total time step, i.e., for the state the considered element has completely passed into a new phase.

It should be noted, that in the process of thermo-mechanical loading, orientation of the axes of the element moving coordinate system may change both as a result of the element quasi-solid motion in the process of inelastic deformation (with the spin tensor) and as a result of the phase transformation in the material. During phase transformation (of a martensitic type), orientation of the element moving coordinate system axes is assumed to be changed almost instantly if the criterion of phase transformation is fulfilled. It means, that if the criterion of a phase transformation was fulfilled in the element at the end of the time step, then it comes to a new time step with a new orientation of the moving coordinate system axes corresponding to its new crystal lattice. In particular, in the martensitic transition the orthogonal tensor, defining 
the saltatory change in orientation of the element moving coordinate system axes as a result of the transformation, can be directly determined from orientation relationships (for example, the Kurdjumov-Sachs ones [27]). This lattice orthogonal transformation is also included into multiplicative decomposition of the transformational deformation gradient under the martensitic transition [25] and ensures existence of an invariant plane.

Thus, if the meso-II element experienced a phase transition at the considered time step, the values of all model internal variables are recalculated for this element at the end of the step (taking into consideration the change of the properties and the element lattice type as a result of phase transformation).

\section{CONCLUSIONS}

he multilevel crystal plasticity model for describing inelastic deformation of polycrystalline materials taking into account the evolving internal material structure is formulated within the paper. The model allows to take into consideration diffusionless solid-state phase transitions of martensitic type. This model, unlike the most existing macro-phenomenological models, provides the opportunity to study evolving material meso- and microstructure. This allows to describe the intense elastoplastic deformations and material's properties after the end of thermo-mechanical processing in detail. Within the presented structure of the model three scale levels are distinguished. They are the macrolevel, the mesolevel-I and the mesolevel-II. The peculiarity of the model is its hybrid character, when the boundary value thermomechanical problem is solved at the macro-level by the finite element method, and lower scale levels are used to consider the material structure applying the statistical modeling methods. The hybrid character of the model determines its relatively low computational costs, allowing to set and solve problems for constructions, not only for material representative volume. Another distinctive feature of the model is that the smallest structural element is so small that it instantly turns into a new martensitic phase. The choice of the martensitic transition variant is based on the thermodynamic criterion of the phase transformation. At the same time, it is not necessary to introduce a huge number of such elements into consideration, the statistical sample under consideration should be a representative volume in the statistical sense, i.e. addition of new elements to the existing ones does not change the current average material properties. In this case, one of the main characteristics determining the anisotropic mechanical and thermo-physical properties of the meso-II element is orientation of the crystallographic coordinate system being abruptly changed as a result of phase transition. The mathematical formulation of the relations describing different modes of inelastic deformation (plastic deformations, lattice rotations, phase transformations), taking into consideration the influence of the external parameters (temperature, applied loading and their rates) and the material characteristics (chemical and phase composition, stacking fault energy, internal stresses due to the defective structure), is provided in the paper. The features of the model implementation algorithm associated with a high rate structure evolution in the phase transition process are given.

\section{ACKNOWLEDGMENTS}

he work was supported by the Russian Science Foundation (grant No. 17-19-01292).

\section{REFERENCES}

[1] Dini, G., Najafizadeh, A., Ueji, R., \& Monir-Vaghefi, S. M. (2010). Improved tensile properties of partially recrystallized submicron grained TWIP steel, Materials Letters, 64(1), pp. 15-18. DOI: 10.1016/j.matlet.2009.09.057

[2] Latypov, M. I., Shin, S., De Cooman, B. C., \& Kim, H. S. (2016). Micromechanical finite element analysis of strain partitioning in multiphase medium manganese TWIP+ TRIP steel, Acta Mater., 108, pp. 219-228. DOI: 10.1016/j.actamat.2016.02.001.

[3] Ding, H., Tang, Z. Y., Li, W., Wang, M., \& Song, D. (2006). Microstructures and mechanical properties of Fe-Mn-(Al, Si) TRIP/TWIP steels, J. Iron. Steel Res. Int., 13(6), pp. 66-70. DOI: 10.1016/S1006-706X(06)60113-1.

[4] Grässel, O., Krüger, L., Frommeyer, G., Meyer, L. W. (2000). High strength Fe-Mn-(Al, Si) TRIP/TWIP steels development-properties-application, Int. J. Plast., 16(10-11), pp. 1391-1409. DOI: 10.1016/S0749-6419(00)00015-2.

[5] Kulawinski, D., Nagel, K., Henkel, S., Hübner, P., Fischer, H., Kuna, M., Biermann, H. (2011). Characterization of stress-strain behavior of a cast TRIP steel under different biaxial planar load ratios, Eng. Fract. Mech., 78(8), pp. 16841695. DOI: 10.1016/j.engfracmech.2011.02.021. 
[6] Roters, F., Eisenlohr, P., Hantcherli, L., Tjahjanto, D. D., Bieler, T. R., \& Raabe, D. (2010). Overview of constitutive laws, kinematics, homogenization and multiscale methods in crystal plasticity finite-element modeling: Theory, experiments, applications, Acta Mater., 58(4), pp.1152-1211. DOI: 10.1016/j.actamat.2009.10.058.

[7] Trusov, P.V., Shveykin, A.I., Nechaeva, E.S., Volegov, P.S. (2012). Multilevel models of inelastic deformation of materials and their application for description of internal structure evolution, Phys. Mesomech., 15(3-4), pp. 155-175. DOI: 10.1134/S1029959912020038.

[8] McDowell, D.L. (2010). A perspective on trends in multiscale plasticity, Int. J. Plast., 26(9), pp. 1280-1309. DOI: $10.1016 /$ j.ijplas.2010.02.008.

[9] Trusov, P.V., Yanz, A.Y. (2016) Physical meaning of nonholonomic strain measure, Phys. Mesomech., 19 (2), pp. $215-$ 222. DOI: $10.1134 / \mathrm{S} 1029959916020156$.

[10] Isupova, I.L., Trusov, P.V. (2013) Review of mathematical models on phase transformations in steels (in Russian), PNRPU Mech. Bull., 3, pp. 157-191.

[11] Tasan, C. C., Diehl, M., Yan, D., Bechtold, M., Roters, F., Schemmann, L., Zheng, C., Peranio, N., Ponge, D., Koyama, M., Tsuzaki, K., Raabe D. (2015). An overview of dual-phase steels: advances in microstructure-oriented processing and micromechanically guided design, Annu. Rev. Mater. Res., 45, pp. 391-431.

DOI: $10.1146 /$ annurev-matsci-070214-021103.

[12] Freidin, A.B., Viltchevskaya, E.N., Sharipova, L. (2002). Two-phase deformations within the framework of phase transition zones, Theor. Appl. Mech. Lett., 28-29, pp. 145-168. DOI: 10.2298/TAM0229145F.

[13] Grinfeld, M.A. (1990). Methods of continuum mechanics in the theory of phase transformations (in Russian), Moscow, Nauka.

[14] Yeddu, H. K., Borgenstam, A., Ågren, J. (2013) Stress-assisted martensitic transformations in steels: A 3-D phase-field study, Acta Mater., 61(7), pp. 2595-2606. DOI: 10.1016/j.actamat.2013.01.039.

[15] Loginova, I., Amberg, G., Agren, J. (2001). Phase-field simulations of nonisothermaly binary alloy solidification., Acta Mater., 49, pp. 573-581. DOI: 10.1016/S1359-6454(00)00360-8.

[16] Trusov, P.V., Shveykin, A.I. (2013). Multilevel crystal plasticity models of single- and polycrystals. Direct models, Phys. Mesomech., 16(2), pp. 99-124. DOI: 10.1134/S1029959913020021.

[17] Trusov P.V., Shveykin A.I. (2013). Multilevel crystal plasticity models of single- and polycrystals. Statistical models, Phys. Mesomech., 16(1), pp. 17-28. DOI: 10.1134/S1029959913010037.

[18] Trusov, P.V., Shveykin, A.I., Yanz, A.Yu. (2017). Motion decomposition, frame-indifferent derivatives, and constitutive relations at large displacement gradients from the viewpoint of multilevel modeling, Phys. Mesomech., 20(4), pp. 357376. DOI:10.1134/S1029959917040014.

[19] Trusov, P.V., Shveykin, A.I. (2017). On motion decomposition and constitutive relations in geometrically nonlinear elastoviscoplasticity of crystallites, Phys. Mesomech., 20(4), pp. 377-191. DOI:10.1134/S1029959917040026.

[20] Shveykin, A.I., Trusov, P.V. (2018) Correlation between geometrically nonlinear elastoviscoplastic constitutive relations formulated in terms of the actual and unloaded configurations for crystallites, Phys. Mesomech., 21(3), pp. 193-202. DOI: 10.1134/S1029959918030025.

[21] Trusov, P.V., Shveykin, A.I., Kondratev, N.S. (2017). Multilevel metal models: Formulation for large displacement gradients, Int. J. Nanomech. Sci. Tech., 8(2), pp. 133-166. DOI: 10.1615/NanoSciTechnolIntJ.v8.i2.40.

[22] Shveykin, A.I., Ashikhmin, V.N., Trusov, P.V. (2010) About models of lattice rotation by metal deformation (in Russian), PNRPU Mech. Bull., 1, pp. 111-127.

[23] Asaro, R. J., Needleman, A. (1985). Overview no. 42 Texture development and strain hardening in rate dependent polycrystals, Acta Metall., 33(6), pp. 923-953. DOI: 10.1016/0001-6160(85)90188-9.

[24] Turteltaub, S., Suiker, A.S.J. (2006). A multiscale thermomechanical model for cubic to tetragonal martensitic phase transformations, Int. J. Solids Struct., 43(14-15), 4509-4545. DOI: 10.1016/j.ijsolstr.2005.06.065.

[25]Nyashina, N.D., Trusov, P.V. (2014). Modelling of martensitic transformations in steels: kinematics of the meso-level (in Russian), PNRPU Mech. Bull., 4, pp. 118-151. DOI: 10.15593/perm.mech/2014.4.05.

[26] Nyashina, N.D. (2015) Mathematical model of the steel deformation at martensitic transformation (in Russian), PNRPU Appl. Math. Cont. Sci., 1, pp. 36-46.

[27] Bhadeshia, H., Honeycombe, R. (2017) Steels: Microstructure and Properties, Amsterdam, Elsevier.

[28] Kurdyumov, G.V., Utevskij, L.M., Entin, R.I. Transformations in iron and steel (in Russian), Moscow, Nauka.

[29] Ko1stinen, D. P., Marbürger, R. E. (1959). A General Equation Prescribing Extent of Austenite-Martensite Transformation in Pure Fe-C Alloy and Plain Carbon Steels. Acta Metall., 7(1), pp. 59-60.

DOI: 10.1016/0001-6160(59)90170-1. 
[30] Cherkaoui, M., Berveiller, M., Sabar, H. (1998). Micromechanical modeling of martensitic transformation induced plasticity (TRIP) in austenitic single crystals, Int. J. Plasticity., 14(7), pp.597-626.

DOI: 10.1016/S0749-6419(99)80000-X

[31] Cherkaoui, M., Berveiller, M., Lemoine, X. (2000). Couplings between plasticity and martensitic phase transformation: overall behavior of polycrystalline TRIP steels, Int. J. Plasticity, 16 (10-11), pp.1215-1241.

DOI: $10.1016 /$ S0749-6419(00)00008-5.

[32] Fischer, F.D., Reisner, G., Werner, E., Tanaka, K., Cailletaud, G., Antretter, T. (2000). A new view on transformation induced plasticity (TRIP), Int. J. Plasticity, 16 (7-8), pp. 723-748. DOI: 10.1016/S0749-6419(99)00078-9.

[33] Grujicic, M., Zhang, Y. (2000). Crystal plasticity analysis of stress-assisted martensitic transformation in Ti-10V-2Fe3Al(wt.\%), J. Mater. Sci., 35(18), pp. 4635 - 4647. DOI: 10.1023/A:1004826301287.

[34] Thamburaja, P., Anand, L. (2001) Polycrystalline shape-memory materials: effect of crystallographic texture, J. Mech. Physics Solids., 49(4), pp.709-737. DOI: 10.1016/S0022-5096(00)00061-2

[35] Iwamoto, T. (2004). Multiscale computational simulation of deformation behavior of TRIP steel with growth of martensitic particles in unit cell by asymptotic homogenization method, Int. J. Plasticity., 20(4-5), pp.841-869. DOI: $10.1016 /$ j.ijplas.2003.05.002.

[36] Olson, G.B., Cohen, M. (1975). Kinetics of strain-induced martensitic nucleation, Metall. Trans. A., 6(4), pp.791-795. DOI: $10.1007 / \mathrm{BF} 02672301$

[37] Lagoudas, D.C., Entchev, P.B. (2004). Modeling of transformation-induced plasticity and its effect on the behavior of porous shape memory alloys. Part I: constitutive model for fully dense SMAs, Mech. Mater., 36(9), pp. 865-892. DOI: $10.1016 /$ j.mechmat.2003.08.006.

[38] Lagoudas, D.C., Entchev, P.B., Popov, P., Patoor, E., Brinson, L.C. Gao, X. (2006). Shape memory alloys. Part II: Modeling of polycrystals, Mech. Mater., 2006, 38(5-6), pp. 430-462. DOI: 10.1016/j.mechmat.2005.08.003.

[39] Patoor E., Lagoudas D.C., Entchev P.B., Brinson L.C., Gao X. (2006). Shape memory alloys, Part I: General properties and modeling of single crystals, Mech. Mater., 38 (5-6), pp.391-429. DOI: 10.1016/j.mechmat.2005.05.027

[40] Van Rompaey, T., Lani, F., Jacques, P.J., Blanpain, B., Wollants, P., Pardoen, T. (2006). Three-dimensional computational-cell modeling of the micromechanics of the martensitic transformation in transformation-inducedplasticity-assisted multiphase steels, Metall. Mater. Trans. A, Vol. 37(1), pp.99-107. DOI: 10.1007/s11661-006-0156-1

[41] Tjahjanto, D.D., Turteltaub, S., Suiker, A.S.J. (2008). Crystallographically based model for transformation-induced plasticity in multiphase carbon steels, Continuum Mech. Thermodyn., 19(7), pp.399-422. DOI: $10.1007 /$ s00161-007-0061-x.

[42] Kouznetsova, V.G., Balmachnov, A., Geers, M.G.D. (2009). A multi-scale model for structure-property relations of materials exhibiting martensite transformation plasticity, Int. J. Mater. Form., 2(1), pp.491-494. DOI: $10.1007 /$ s12289-009-0578-6.

[43] Kouznetsova, V.G., Geers, M.G.D. (2008). A multi-scale model of martensitic transformation plasticity, Mech. Mater., 40(8), pp. 641-657. DOI: 10.1016/j.mechmat.2008.02.004.

[44] Sengupta A., Papadopoulos P., Taylor-R.L. (2009). Multiscale finite element modeling of superelasticityin Nitinolpolycrystals, Comput. Mech., 43(5), pp.573-584. DOI: 10.1007/s00466-008-0331-x.

[45] Isupova, I.L., Trusov, P.V. (2013) Mathematical modeling of phase transformations in steel under thermomechanical loading (in Russian), PNRPU Mech. Bull., 3, pp. 127-157.

[46] De Groot, S.R. (1951). Thermodynamics of irreversible processes, Amsterdam, North-Holland Publishing Company.

[47] Kondepudi, D., Prigogine, I. (2015). Modern thermodynamics. From heat engines to dissipative structures, Chichester, Wiley.

[48] Trusov, P.V., Isupova, I.L. (2014) Two-scale model of thermomechanically loaded steel with martensite transformations (in Russian), Phys. Mesomech., 17(2), pp. 5-17.

[49] Miettinen, J. (1997). Calculation of solidification-related thermophysical properties for steels, Metall. Mater. Trans B., 28(2), pp. 281-297. DOI: 10.1007/s11663-997-0095-2.

[50] Redlich, O., Kister, A.T. (1948). Algebraic representation of thermodynamic properties and the classification solutions, Ind. Eng. Chem., 40(2), pp.345-348. DOI: 10.1021/ie50458a036.

[51] Dinsdale, A.T. (1991). SGTE Data for Pure Elements, Calphad., 15(4), pp.317-425. DOI: $10.1016 / 0364-5916(91) 90030-N$.

[52] Nechaeva, E.S., Trusov, P.V. (2011). Constitutive model of semicrystalline polymer material. implementation algorithm for mezolevel model (in Russian), Comp. Cont. Mech., 4(1), pp. 74-89. 